\title{
Estimation of State of Charge of Lithium-Ion Batteries Based on Wide and Deep Neural Network Model
}

\author{
Di Mu $(\mathbb{D})$ and Shuning Wang $(\mathbb{D}$ \\ Changzhou Power Supply Branch of State Grid Jiangsu Electric Power Co. Ltd., Changzhou, Jiangsu Province, China \\ Correspondence should be addressed to Di Mu; 1921783267@qq.com
}

Received 15 October 2021; Accepted 22 November 2021; Published 9 December 2021

Academic Editor: Gianpaolo Di Bona

Copyright (c) 2021 Di Mu and Shuning Wang. This is an open access article distributed under the Creative Commons Attribution License, which permits unrestricted use, distribution, and reproduction in any medium, provided the original work is properly cited.

\begin{abstract}
It is important to accurately estimate the SOC to ensure that the lithium-ion battery is within a safe working range, prevent overcharging and over-discharging, and ultimately improve battery life. However, SOC is an internal state of the battery and cannot be directly measured. This paper proposes a SOC estimation method based on the wide and deep neural network model, which combines the linear regression (LR) model and the backpropagation neural network (BPNN) model. This article uses the dataset provided by the Advanced Energy Storage and Applications (AESA) group to verify the performance of the model. The performance of the proposed model is compared with the common BPNN model in terms of root mean square error (RMSE), average absolute proportional error (MAPE), and SOC estimation error. The validation results prove that the effect of the proposed model in estimating SOC is better than that of the ordinary BPNN model. Compared with the BPNN model, the RMSE values of the SOC predicted value of the wide and deep model in the charging and discharging stages were reduced by $10.2 \%$ and $15.4 \%$, respectively. Experimental results show that the maximum SOC estimation error of the model in predicting the SOC during charging and discharging is $0.42 \%$ and $0.86 \%$, respectively.
\end{abstract}

\section{Introduction}

With the massive use of fossil fuels, environmental pollution and energy shortages have become increasingly serious. Governments are paying more and more attention to the development and utilization of clean energy. In recent years, electric vehicles have received great attention from the government and enterprises [1]. Different types of electric vehicles have emerged in the market, such as pure electric vehicles (BEVs), hybrid electric vehicles (HEVs), fuel cell vehicles (FCEVs), and so on [2]. Rechargeable batteries are widely used in electric vehicles, such as lead-acid batteries, nickel-cadmium batteries, nickel-hydrogen batteries, and lithium-ion batteries. Among them, lithium-ion batteries are favored by many companies due to their advantages such as long life, low pollution, high power, and fast charging, and its market proportion is also constantly increasing [3].

Therefore, battery management technology has attracted much attention in recent years. In [4], the authors proposed an enabling state of health $(\mathrm{SOH})$ estimation scheme based on the ICA method for real-world EVs. This is realized by combining an equivalent IC-value calculation for battery packs with cell-level battery tests while taking cell inconsistency into consideration. In [5], the authors proposed a multistage alternative current (AC) strategy for internally hearting lithium-ion batteries. In [6], a data-driven method was proposed for battery charging capacity diagnosis based on massive real-world EV operating data.

The estimation of state of charge (SOC) of lithium-ion batteries is also a key part of battery management technology. Lithium-ion batteries will age, that is, their capacity will gradually decay as the number of uses increases. There are many reasons for the accelerated decay rate of lithiumion battery capacity, including excessive temperature, overcharging, over-discharging, and not using it for a long time after being fully charged [7]. To prevent over-charging and over-discharging, there must be an accurate estimation of the SOC of the lithium-ion battery.

The state of charge (SOC) is a relative measure of the energy stored in a battery, defined as the ratio of the amount 
of charge that can be extracted from a battery cell at a specific point in time to the rated capacity [8].The calculation formula of SOC can be expressed as

$$
\mathrm{SOC}=\frac{Q_{c}}{C_{N}},
$$

where $Q_{c}$ is the remaining capacity of the battery and $C_{N}$ is the rated capacity. It is important to accurately estimate the SOC to ensure that the lithium-ion battery is within a safe working range, prevent over-charging and over-discharging, and ultimately improve battery life. Because the capacity that the battery can release is affected by many factors, including discharge rate, discharge current, battery internal temperature, self-discharge, charge and discharge cycles, battery aging, and so on, the SOC must be related to these factors.

There are many traditional methods for SOC estimation, including the coulomb counting method $[9,10]$, open circuit voltage method [11], Kalman filtering method, and so on $[12,13]$. The coulomb counting method is the most commonly used SOC estimation method. If initial state is $\mathrm{SOC}_{0}$, then the current SOC is

$$
\mathrm{SOC}=\mathrm{SOC}_{0}-\frac{1}{C_{N}} \int_{0}^{t} \eta I \mathrm{~d} t,
$$

where $I$ is the battery current and $\eta$ is the efficiency of charging and discharging. The shortcomings of this method are as follows: inaccurate current measurement will cause SOC calculation errors, which will accumulate for a long time, and the error will become larger and larger; the battery charging and discharging efficiency must be considered; and the error will be larger under the condition of high temperature and severe current fluctuations. The open circuit voltage of the battery is close to the electromotive force of the battery in value. There is a relatively fixed functional relationship between the open circuit voltage and the SOC [14], so that the SOC can be estimated based on the open circuit voltage. The obvious disadvantage is that the battery needs to be left to stand for a long time to achieve voltage stability [11]. It takes several hours to restore the battery state from work to stability, which causes difficulties in measurement. How to determine the resting time is also a problem, so the method is only suitable for the parking state of electric vehicles. The open circuit voltage method is effective in estimating SOC in the initial and final stages of charging and is often used in combination with the coulomb counting method. The core idea of Kalman filter theory is to make an optimal estimation of the state of the dynamic system in the sense of minimum variance. This method is suitable for all kinds of batteries. Compared with other methods, it is especially suitable for the estimation of the battery SOC of hybrid electric vehicles with severe current fluctuations. It not only gives the estimated value of SOC but also gives the estimation error of SOC.

With the rapid development of machine learning technology in recent years, more and more researchers have begun trying to use data-driven methods to make predictions about SOC. These methods can automatically learn network parameters through intelligent algorithms and obtain the relationship between battery parameters and SOC from them $[15,16]$. Machine learning methods commonly used for SOC estimation include neural networks [17], support vector machine (SVM) [18], extreme learning machine (ELM) [19], and so on. Recent neural network structures for SOC estimation mainly include wavelet neural network (WNN) [20, 21], backpropagation neural network (BPNN) [22], radial basis function neural network (RBFNN) [23], and so on. In [24], the authors combined adaptive wavelet neural networks with a discrete wavelet transform, proposing a new hybrid wavelet neural network model based on the Levenberg-Marquardt algorithm to estimate the SOC. In [25], an improved BPNN-based SOC estimation method for lithiumion batteries was proposed. The method used two algorithms: principal component analysis and particle group optimization, to improve the accuracy and robustness of the model.

Neural network has strong expression ability and generalization ability but lacks "memory ability." "Memory ability" can be understood as the ability of the model to directly learn and use the "co-occurrence frequency" of items or features in historical data. The linear model has strong memory ability. This article uses the wide and deep model to estimate the SOC. The wide and deep model is a hybrid model composed of a singlelayer wide part and a multilayer deep part [26]. Among them, the wide part is a single-layer linear model whose main function is to make the model have the "memory ability," and the deep part is the BPNN model whose main function is to make the model have generalization ability. It is this structural feature that makes the model have the advantages of both linear model and deep neural network. Therefore, the wide and deep model can swiftly learn and memorize a large number of historical behavior characteristics while also having great expressive ability.

The rest of this article is organized as follows. In Section 2 , the principle and basic structure of the wide and deep model are introduced in detail, and how to use it for SOC prediction is also given. In Section 3, the method of obtaining training data and the original data analysis are introduced. Section 4 gives the experimental results and analysis. Section 5 draws the conclusion.

\section{Wide and Deep Model}

The wide and deep model connects the wide part of the single input layer with the deep part, which is composed of the embedding layer and multiple hidden layers, and then inputs them to the final output layer. The wide part is good at handling a large number of sparse category features. The deep part uses the strong expressive ability of the neural network to mine the data patterns hidden behind the features. Finally, using the linear regression model, the output layer combines the wide part and the deep part to form a unified model, as shown in Figure 1.

2.1. Wide Part. The wide part is a generalized linear model, which can be expressed as follows:

$$
y=w^{T} x+b
$$

where $y$ is the predictive value; $w^{T}=\left[w_{1}, w_{2}, \ldots, w_{n}\right]$ is the weight of features; $x=\left[x_{1}, x_{2}, \ldots, x_{n}\right]^{T}$ is the feature vector; and $b$ is the bias. 


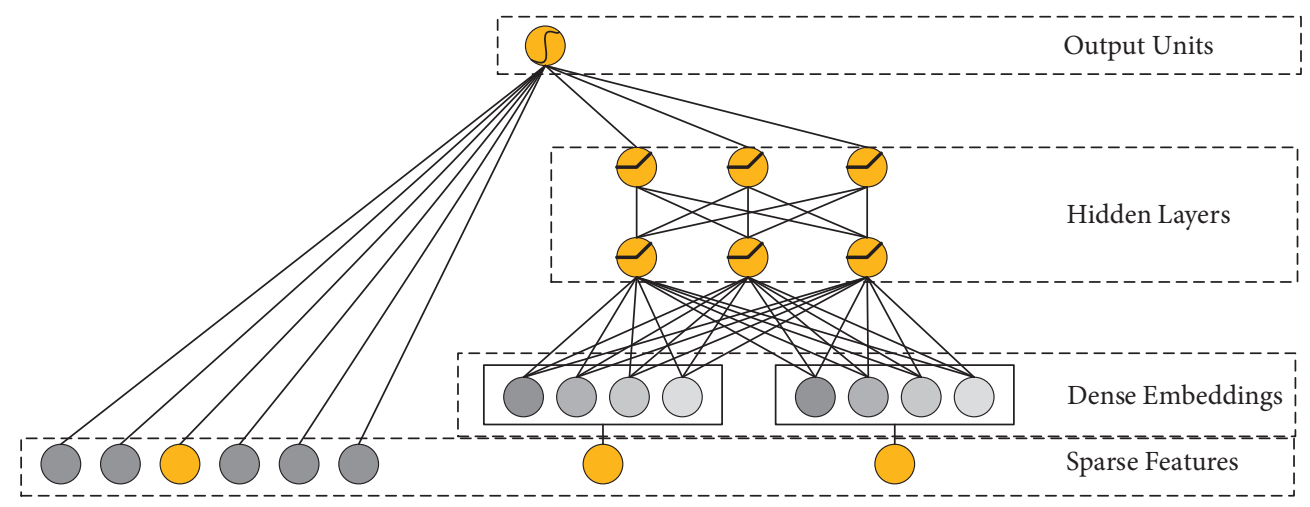

FIGURE 1: Schematic diagram of the wide and deep model.

The features utilized in the wide part are usually original features or new features made by applying some simple transformations to the original features. Cross-product transformation is the most common transformation method employed, and it is typically applied to category features. Table 1 shows the data of a group of users watching videos. The original data have only two features: user id: $\left\{u_{1}, u_{2}, u_{3}\right\}$ andvideo id: $\left\{f_{1}, f_{2}\right\}$. After one-hot encoding, the dimensions of these two features are 3 and 2, respectively, and the corresponding cross-product features of these two features are user id $\times$ video id: $\left\{u_{1} \wedge f_{1}, u_{1} \wedge f_{2}, u_{2} \wedge f_{1}, u_{2} \wedge f_{2}, u_{3} \wedge f_{1}\right.$, $\left.u_{3} \wedge f_{2}\right\}$, with a dimension of 6 . The nonlinear features obtained by the cross-product transformation can capture the high-order correlation between the original features. If the original dense feature is to be subjected to cross-product transformation, it needs to be discretized.

The advantage of the wide model with cross-product features is that the model has high interpretability and the feature importance is easy to analyse. There are three main disadvantages. One is that a lot of manual design is required. The other is that if the original category feature dimension is too high, then the feature dimension obtained through cross-product transformation will be very large, which will cause over-fitting problems. The solution is to construct more coarse-grained cross features. The third is that when the number of original features $n$ is too large, in order to obtain high-order correlation between features, the number of cross-product features that need to be constructed is $O\left(n^{2}\right)$, which is basically impossible. At this time, a deep neural network needs to be used.

2.2. Deep Part. The deep part is a backpropagation neural network, which consists of three components: input layer, hidden layer, and output layer. The input features generally include sparse and dense features. The dense features can be input directly. The sparse feature must be encoded into a vector form before input. A simple one-hot encoding method can be employed when the sparse feature dimension is low. When the sparse feature dimension is too high, the use of onehot encoding will cause the problem of dimensional explosion. At this time, the sparse feature can be trained into a lowdimensional word vector.
After the feature is input, it will enter the hidden layer, and each neuron in the hidden layer will perform the following operations:

$$
x_{i}^{l+1}=f\left(W_{i}^{l} x^{l}+b_{i}^{l}\right),
$$

where $W_{i}^{l}, b_{i}^{l}$ are the weight and bias of the $i$-th neuron in the $l$-th hidden layer; $x^{l}$ is the activation value of the $l$-th hidden layer, and its dimension is equal to the number of neurons in the $l$-th layer; $x_{i}^{l+1}$ is the $i$-th activation value of the $l+1$-th hidden layer; and $f$ is the activation function. This article uses the Relu function as the activation function, and its function expression is as follows:

$$
f(x)=\max (0, x) .
$$

The deep part can make up for the defects of the wide part. The deep part does not require much manual participation, but it can have better generalization of the cross features that the wide part cannot construct.

2.3. Joint Part. The joint part uses the log-weighted sum of the output of the wide layer and the output of the deep layer as the final predicted value. The calculation formula is as follows:

$$
y_{p}=\sigma\left(w_{\text {wide }}^{T} x_{\text {wide }}+w_{\text {deep }}^{T} x_{\text {deep }}^{l f}+b^{l f}\right)
$$

where $\sigma$ is the sigmoid function; $w_{\text {wide }}^{T}$ is the weight of the wide layer; $w_{\text {deep }}^{T}$ is the weight used for the final activation value of the deep layer; and $x_{\text {deep }}^{l f}$ is the activation value of the last hidden layer.

The model selects logistic loss as the loss function, and the loss is calculated as follows:

$$
J=-\frac{1}{m}\left[\sum_{i=1}^{m} y^{(i)} \log y_{p}^{(i)}+\left(1-y^{(i)}\right) \log \left(1-y_{p}^{(i)}\right)\right]+\Omega,
$$

where $m$ is the number of training samples; $y^{(i)}, y_{p}^{(i)}$ are the true value and predicted value of the $i$-th sample, respectively; and $\Omega$ is the regularization term. The weights and biases need to be updated according to the loss. Using AdaGrad as the optimizer of the model, the calculation method is as follows: 
TABle 1: User-video data.

\begin{tabular}{cccccc}
\hline & Userid & Userid-one-hot & Videoid & Videoid-one-hot & Userid $\times$ videoid \\
\hline 0 & $u_{1}$ & {$[1,0,0]$} & $f_{1}$ & {$[0,1]$} & {$[0,1,0,0,0,0]$} \\
1 & $u_{2}$ & {$[0,1,0]$} & $f_{2}$ & {$[1,0]$} & $0,0,1,0,0,0]$ \\
2 & $u_{3}$ & {$[0,0,1]$} & $f_{1}$ & {$[0,1]$} & {$[0,0,0,0,0,1]$} \\
3 & $u_{3}$ & {$[0,0,1]$} & $f_{2}$ & {$[1,0]$} & {$[0,0,0,0,1,0]$} \\
\hline
\end{tabular}

$$
\begin{aligned}
W_{i, j}^{l}(n+1) & =W_{i, j}^{l}(n)-\frac{\eta}{\sqrt{G_{t}+\varepsilon}} \frac{\partial J}{\partial W_{i, j}^{l}(n)}, \\
b_{i}^{l}(n+1) & =b_{i}^{l}(n)-\frac{\eta}{\sqrt{G_{t}+\varepsilon}} \frac{\partial J}{b_{i}^{l}(n)}, \\
w_{i}(n+1) & =w_{i}(n)-\frac{\eta}{\sqrt{G_{t}+\varepsilon}} \frac{\partial J}{w_{i}(n)},
\end{aligned}
$$

where $\eta$ is the learning rate; $\varepsilon$ is a very small value to prevent the denominator from being 0 ; and $G_{t}$ is the square accumulation of the gradient of the parameter.

2.4. Estimating SOC with the Wide and Deep Model. This article uses the wide and deep model to predict the SOC state value during the charging and discharging process of lithium-ion batteries. The features used are current $i$, voltage $v$, $d v, d i, d^{2} v, d^{2} i$, and the battery charge cycles. All features must be normalized before entering the model. In this experiment, the max-min normalization method was used to normalize the value to $[0,1]$. The formula is as follows:

$$
x_{i}^{\prime}=\frac{x_{i}-x_{\min }}{x_{\max }-x_{\min }},
$$

where $x^{i}$ is the original value; $x_{\min }, x_{\max }$ are the minimum and maximum values of the feature, respectively; and $x_{i}^{\prime}$ is the normalized value.

The input of the wide part includes the original 7 features. For the deep part, the 7 original features were first processed into embedding vectors by PCA and then input into the model. The model structure is shown in Figure 2.

\section{Training and Testing Data}

3.1. Data Sources. In order to verify the performance of the wide and deep model, this paper uses the lithium-ion battery charge and discharge data of the Mendeley dataset of AESA. These data are obtained by charging and discharging two lithium-ion batteries with a standard capacity of $27 \mathrm{Ah}$ for 100 times. When charging, the CC-CV protocol is used. First, the battery is charged with a constant current of $27 \mathrm{~A}$ until the terminal voltage reaches $4.2 \mathrm{~V}$, and then the terminal voltage is kept at $4.2 \mathrm{~V}$ for constant voltage charging until the current drops to 2.7 A to stop charging. When discharging, adopt the CC discharge protocol. Keeping the current at $81 \mathrm{~A}$, stop discharging when the terminal voltage drops to $2.75 \mathrm{~V}$. The above process is repeated 100 times, with an interval of 30 minutes each time, and the ambient temperature is always $40^{\circ} \mathrm{C}$. The overall procedure of the lithium-ion charge and discharge experiment is shown in Figure 3.

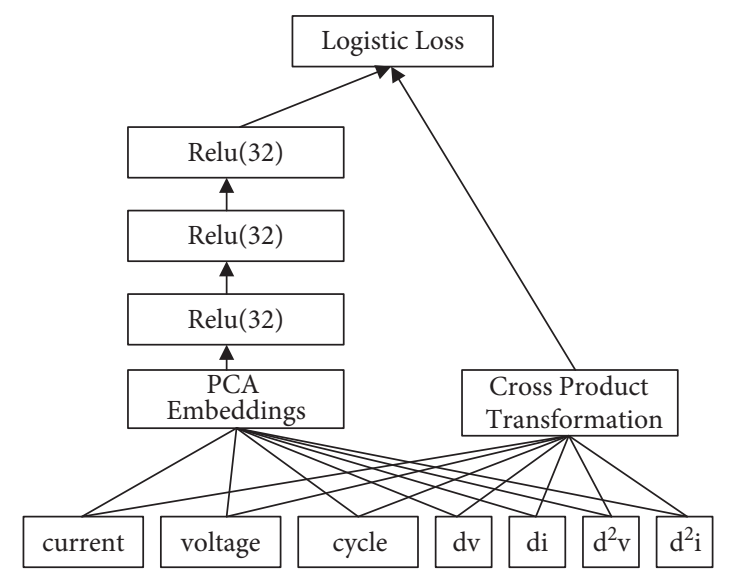

FIGURE 2: Estimating SOC with the wide and deep model.

\subsection{Data Analysis}

3.2.1. Changes of the Battery Maximum Charge Capacity. In practice, the maximum charge capacity of the battery will reduce with the increase in the number of charges. Since the current, voltage, and temperature are strictly controlled during the experiment, over-charging, over-discharging, and the influence of the external environment on the battery capacity are avoided. Therefore, the capacity degradation of the battery in this experiment is mainly caused by the internal factors of the battery. In terms of the electrode, repeated charging and discharging reduce the active surface area of the electrode, the structure of the active material changes, and the electrical contact of the active particles becomes worse. In the electrolyte solution, the electrolyte or the decomposition of conductive salt causes its conductivity to decrease, and the decomposition product causes interface passivation. The change in the charge capacity of the two batteries in this experiment is shown in Figure 4(a). Figure 4(b) shows the difference in capacity between the two batteries. It can be seen that the largest difference in capacity between them is only $0.122 \mathrm{Ah}$. The change in battery capacity is almost the same.

\subsubsection{The Difference between Charge Capacity and Discharge} Capacity. During each round of charging and discharging, the discharge capacity of the lithium-ion battery is often smaller than the charging capacity, as shown in Figures 5 and 6. This is mainly caused by two reasons. One is that during the first few rounds of charging, a solid electrolyte interface (SEI) is formed on the surface of the negative electrode, which consumes lithium ions from the positive electrode. These lithium ions no longer participate in the subsequent 


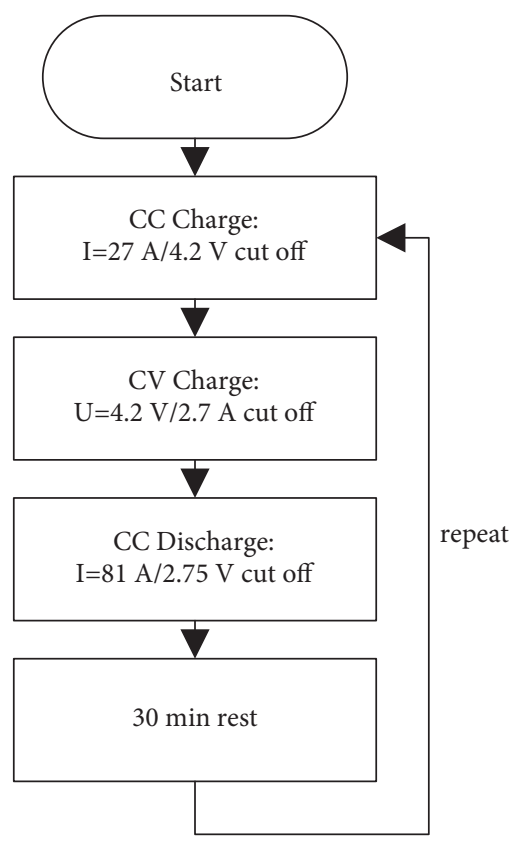

FIgURE 3: The procedure of lithium-ion battery cycle experiment.

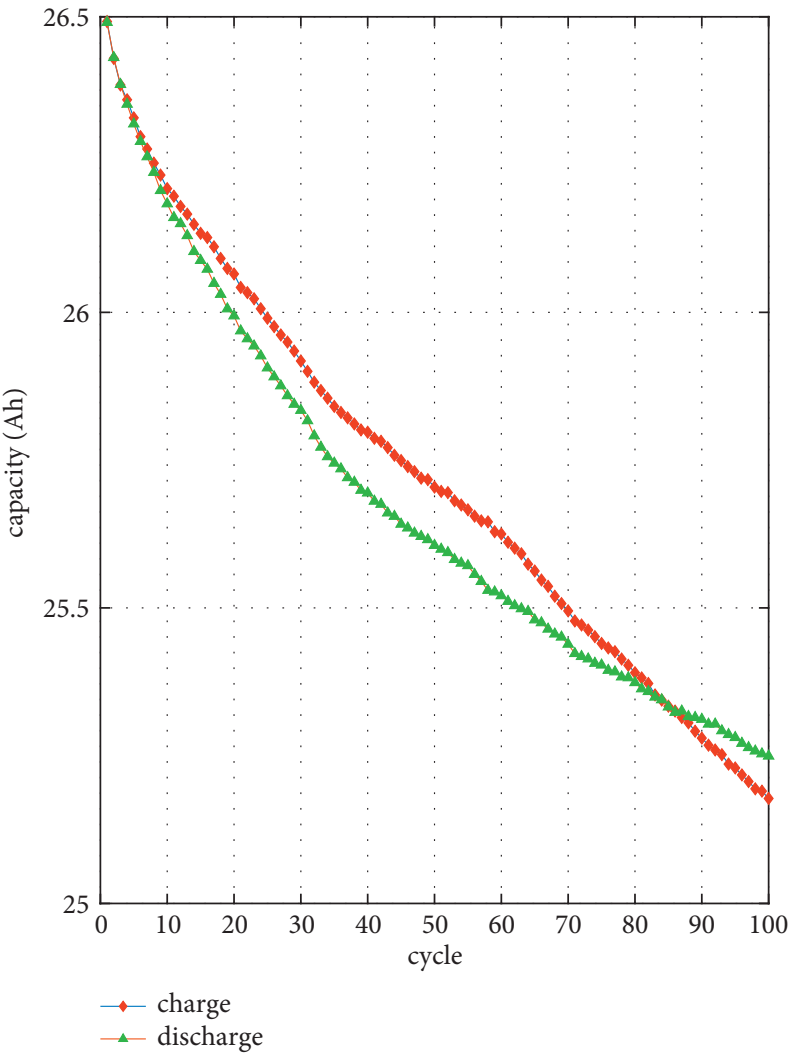

(a)

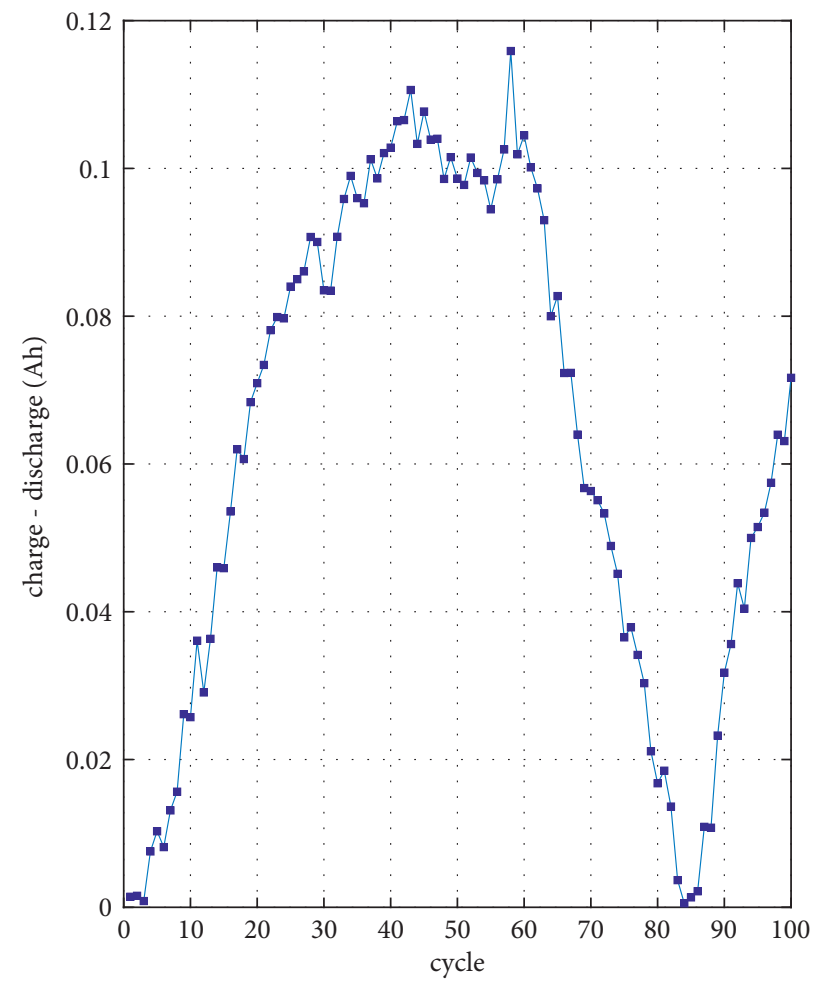

(b)

FIGURE 4: (a) The charge capacity of battery 1 and battery 2 changing with the number of charges. (b) The difference in charge capacity of the two batteries.

discharge process. It can be seen from Figure 5(b) in the figure that the charge and discharge capacity of the previous rounds is quite different. The second is due to the fact that a very small amount of lithium ions cannot be extracted after being inserted into the negative electrode under normal conditions. 


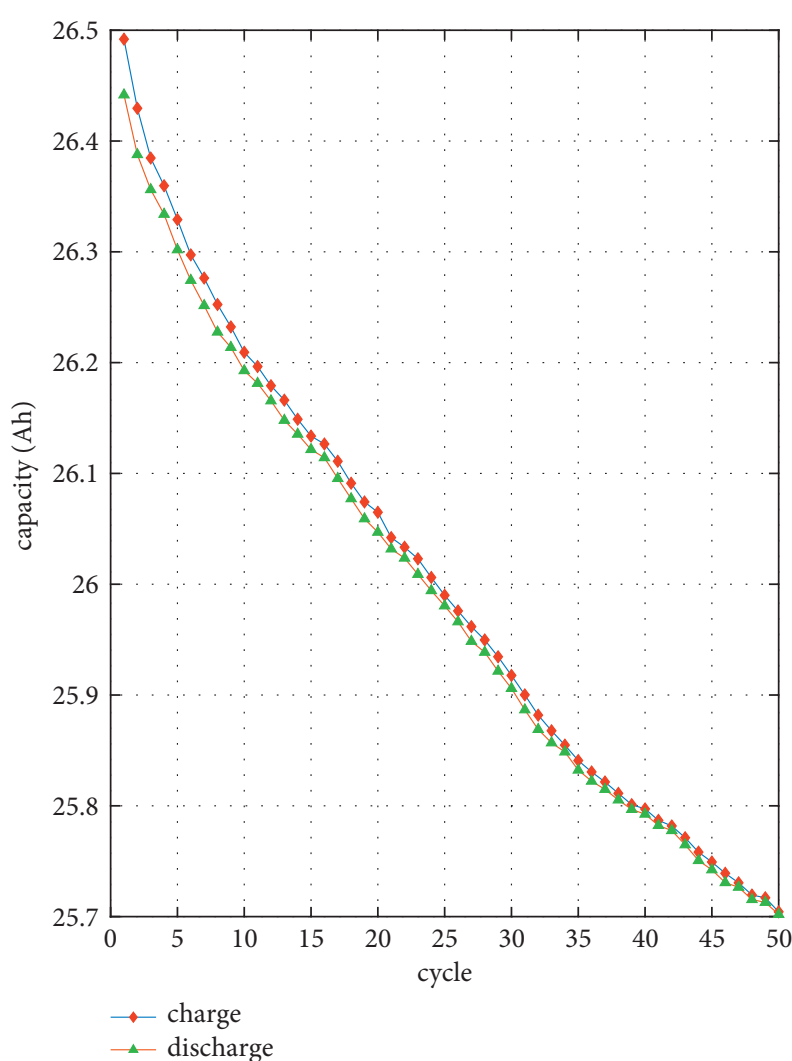

(a)

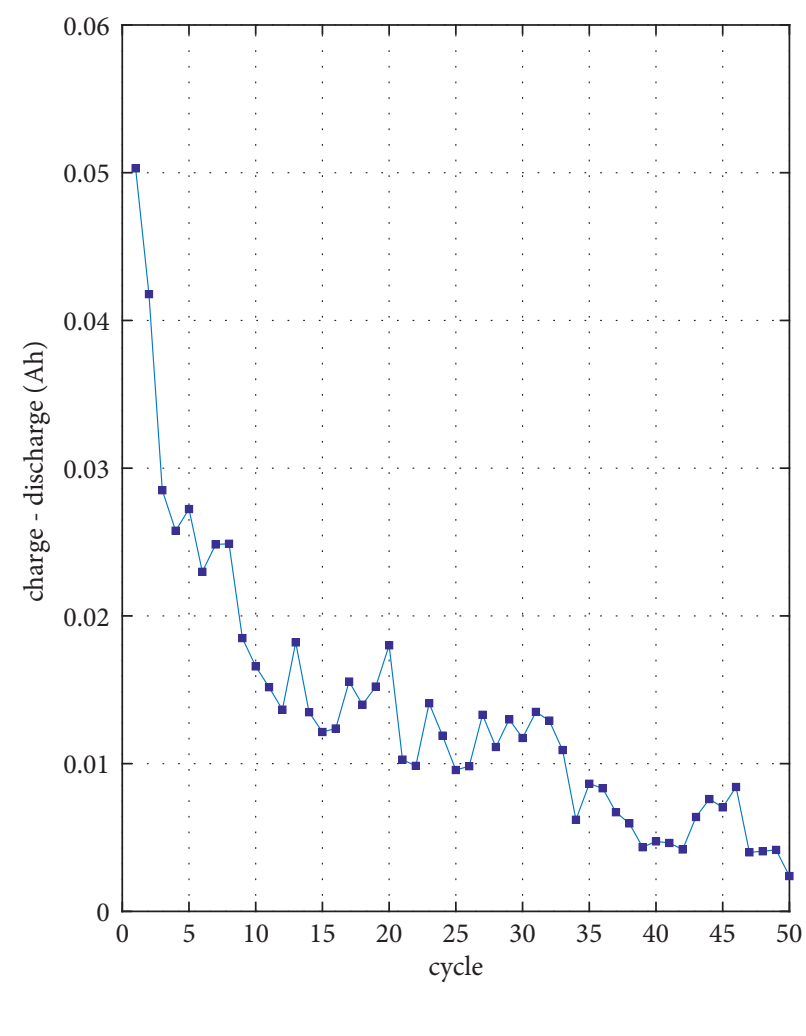

(b)

FIGURE 5: Difference in charge and discharge capacity of battery 1.

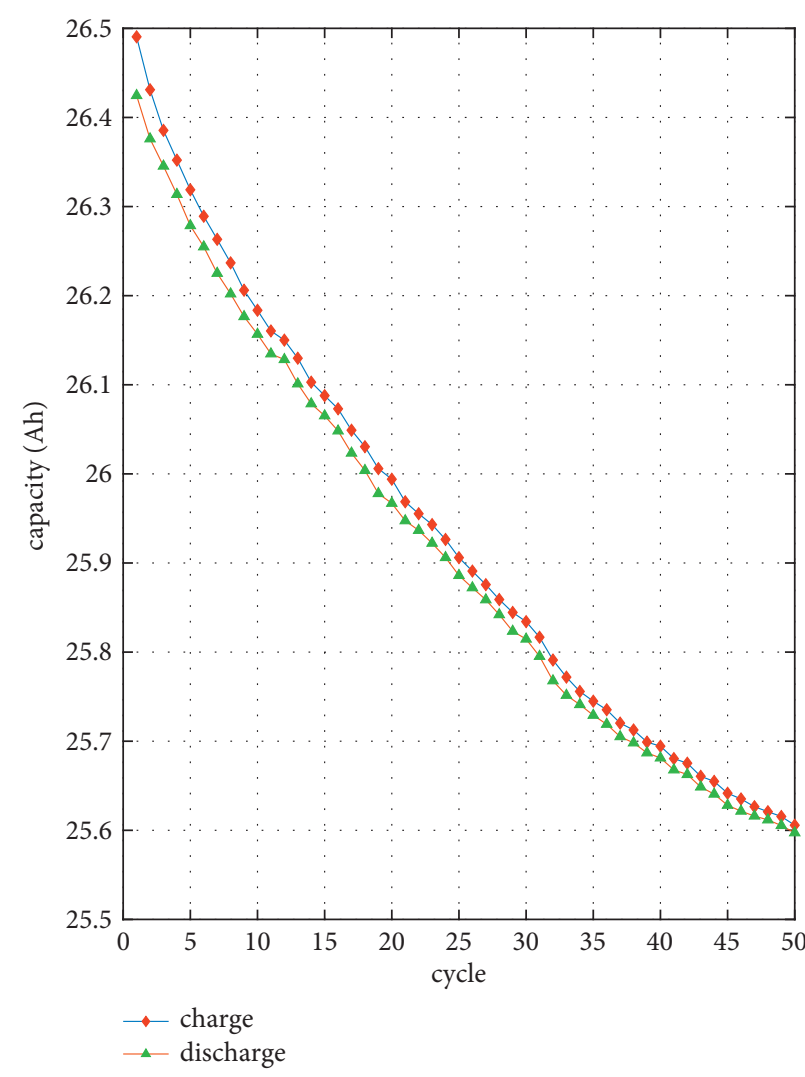

(a)

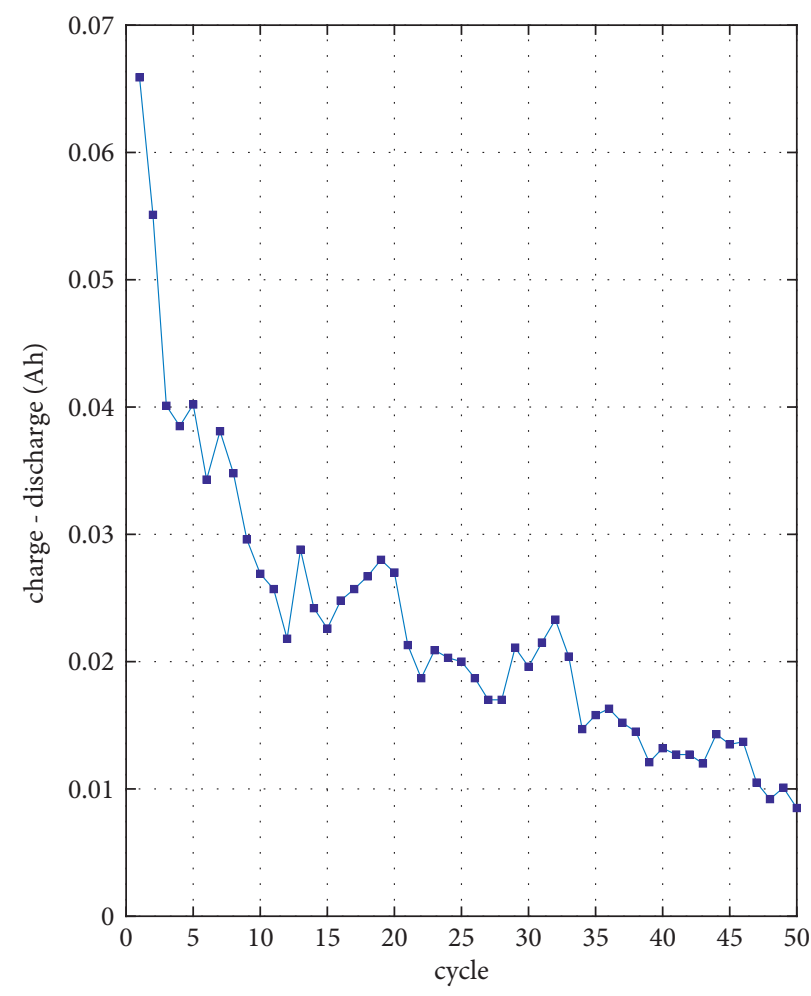

Figure 6: Difference in charge and discharge capacity of battery 2. 


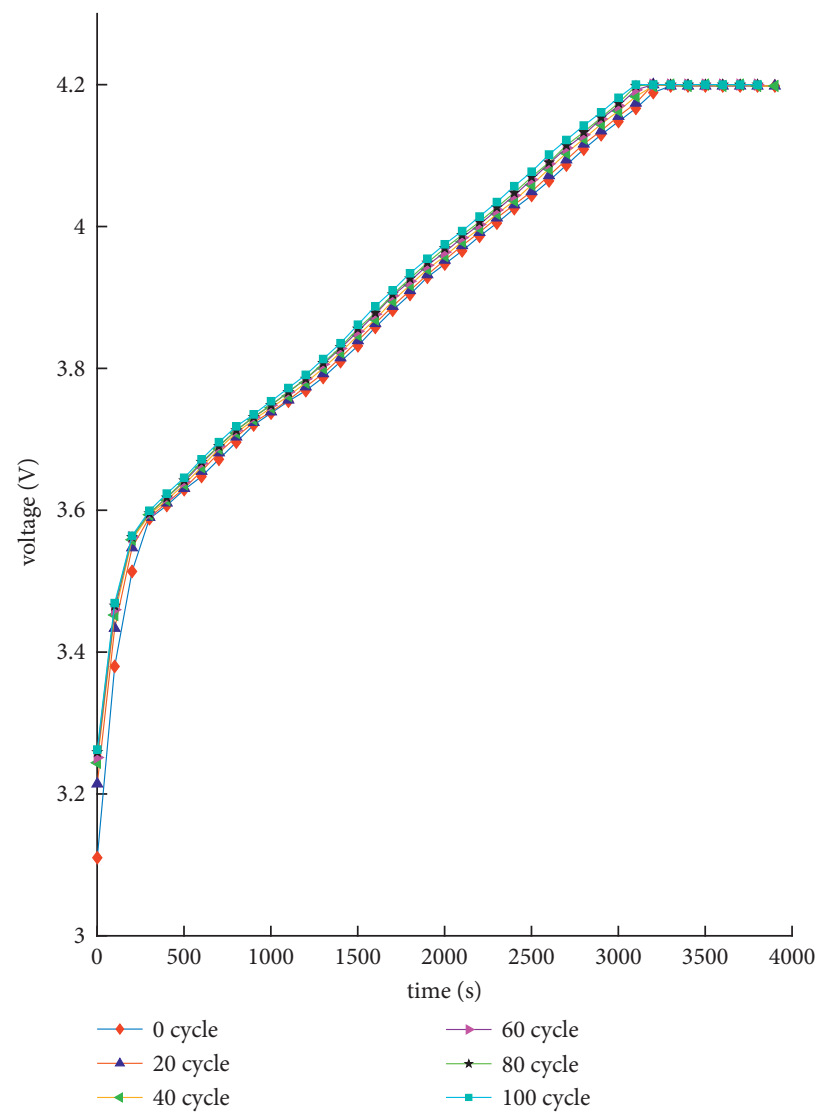

(a)

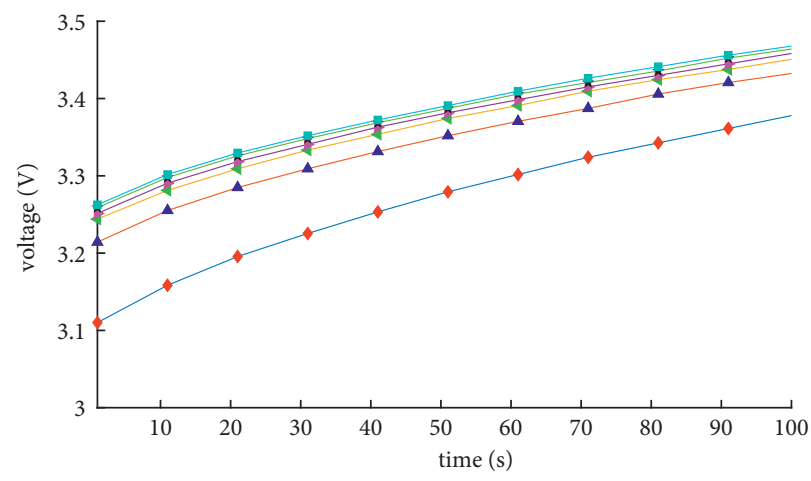

(b)

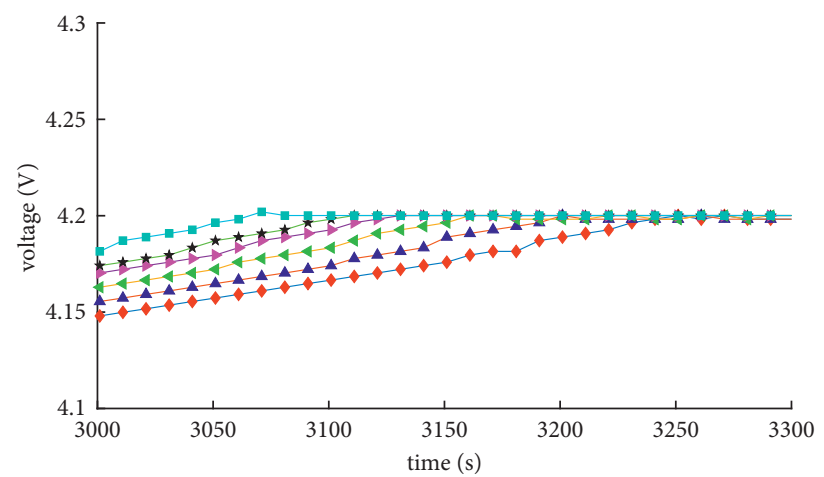

(c)

Figure 7: (a) The change of terminal voltage with time in different rounds. (b, c) The partially enlarged diagrams of (a).

3.2.3. Changes in Current, Voltage, and Charging Capacity over Time. Figure 7 shows the change in the terminal voltage with the charging time in different rounds. It can be clearly seen from Figure $7(\mathrm{c})$ that as the number of charge and discharge increases, the terminal voltage will reach $4.2 \mathrm{~V}$ faster during charging. Figure 8 shows the changes in current and SOC over time after entering constant voltage charging.

\section{Experimental Results and Analysis}

4.1. Performance Indicators. This article uses two statistical errors to verify the performance of the wide and deep model: root mean square error (RMSE) and mean absolute percentage error (MAPE). Their calculation formulas are as follows:

$$
\begin{aligned}
\text { RMSE } & =\sqrt{\frac{1}{N} \sum_{i=1}^{N}\left(\operatorname{soc}_{\text {estimate }}-\operatorname{soc}_{\text {true }}\right)^{2},} \\
\text { MAPE } & =\frac{1}{N} \sum_{i=1}^{N}\left|\frac{\text { soc }_{\text {estimate }}-\operatorname{soc}_{\text {true }}}{\operatorname{soc}_{\text {true }}}\right|,
\end{aligned}
$$

where $\operatorname{soc}_{\text {true }}$, $\operatorname{soc}_{\text {estimate }}$ are the real SOC and the estimated SOC, respectively, and $N$ is the number of samples. 


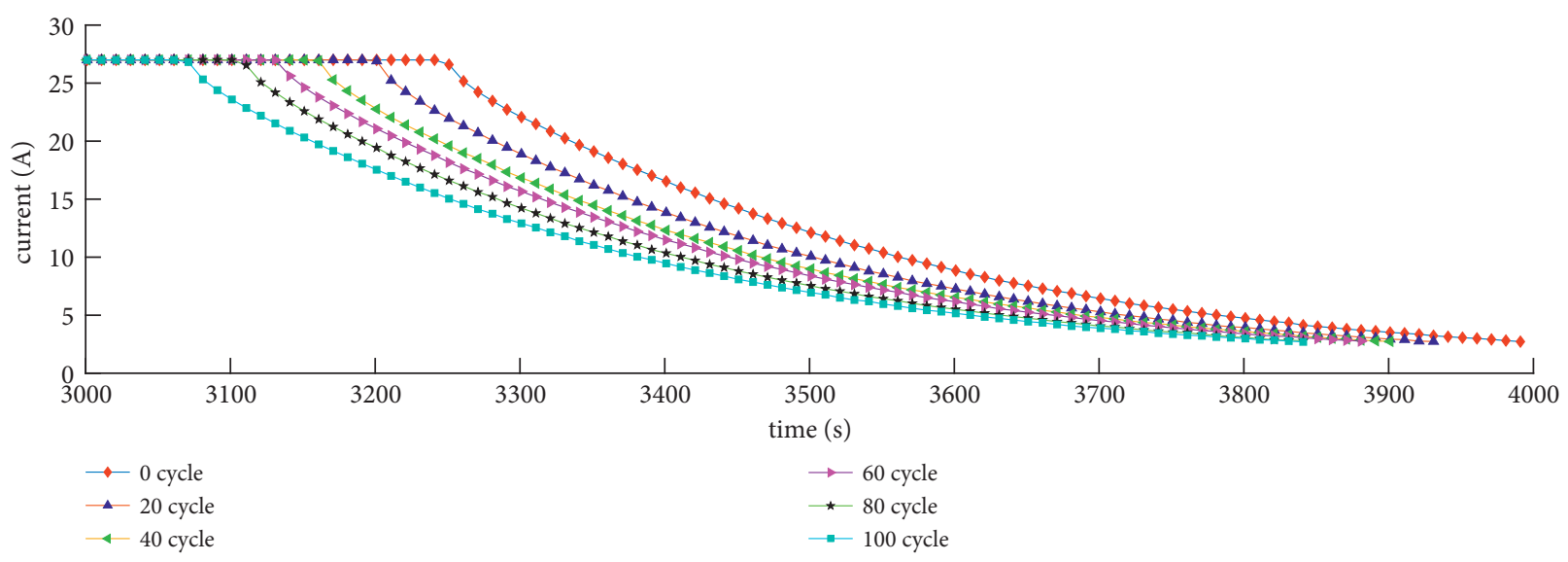

(a)

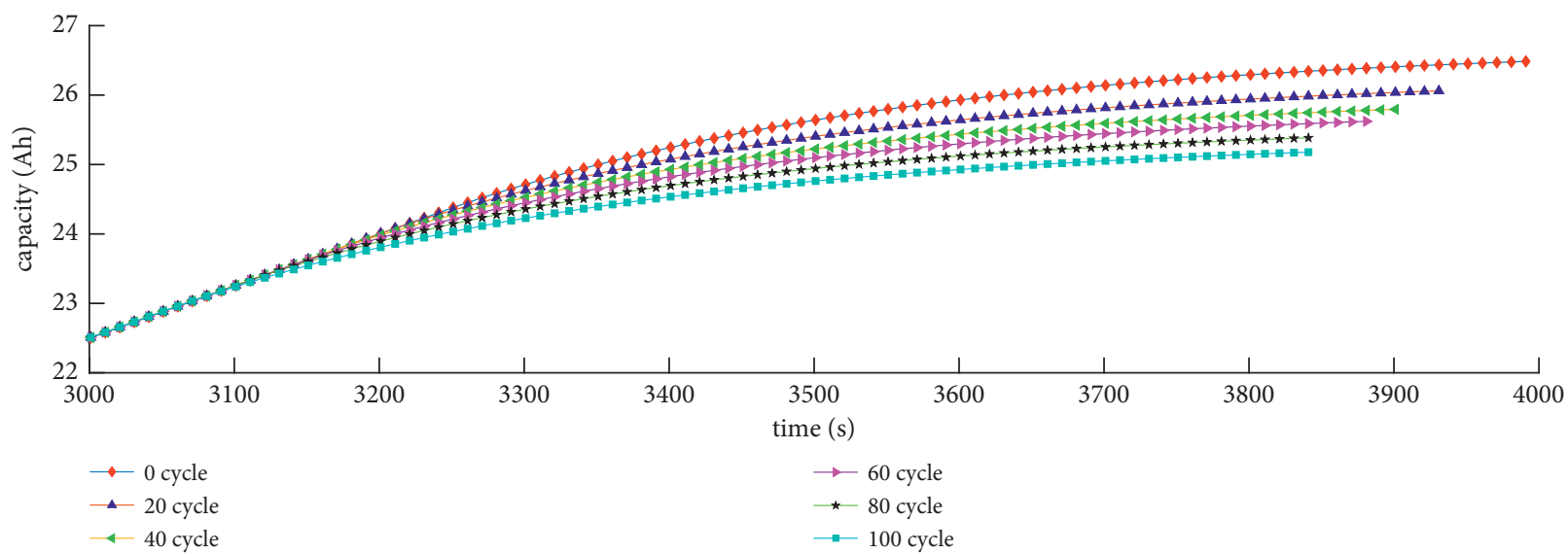

(b)

FIGURE 8: Changes in current (a) and battery SOC (b) with time in different rounds.

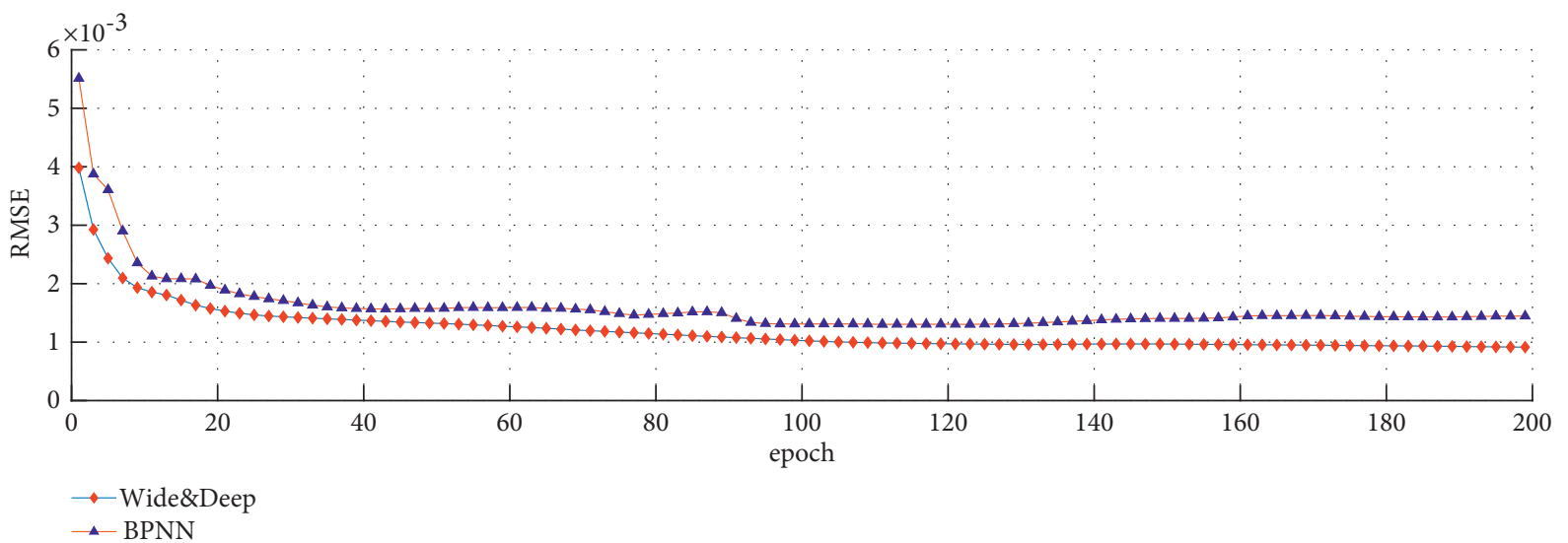

(a)

Figure 9: Continued. 


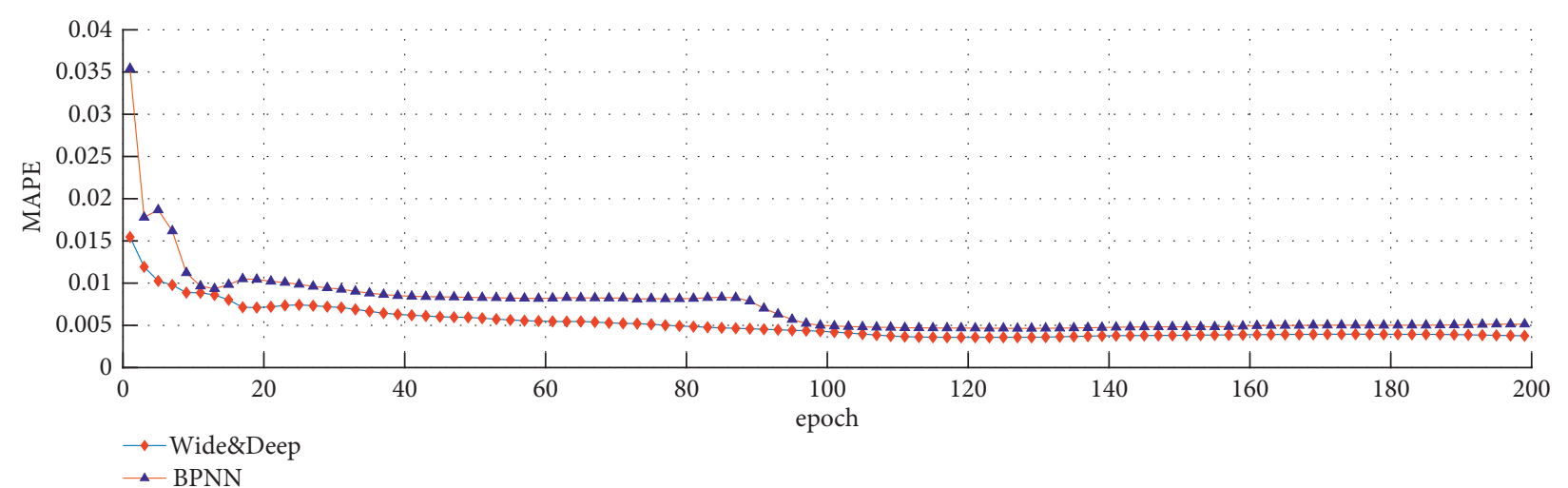

(b)

FIGURE 9: RMSEs and MAPEs of wide and deep model and BPNN model with respect to the number of epochs.

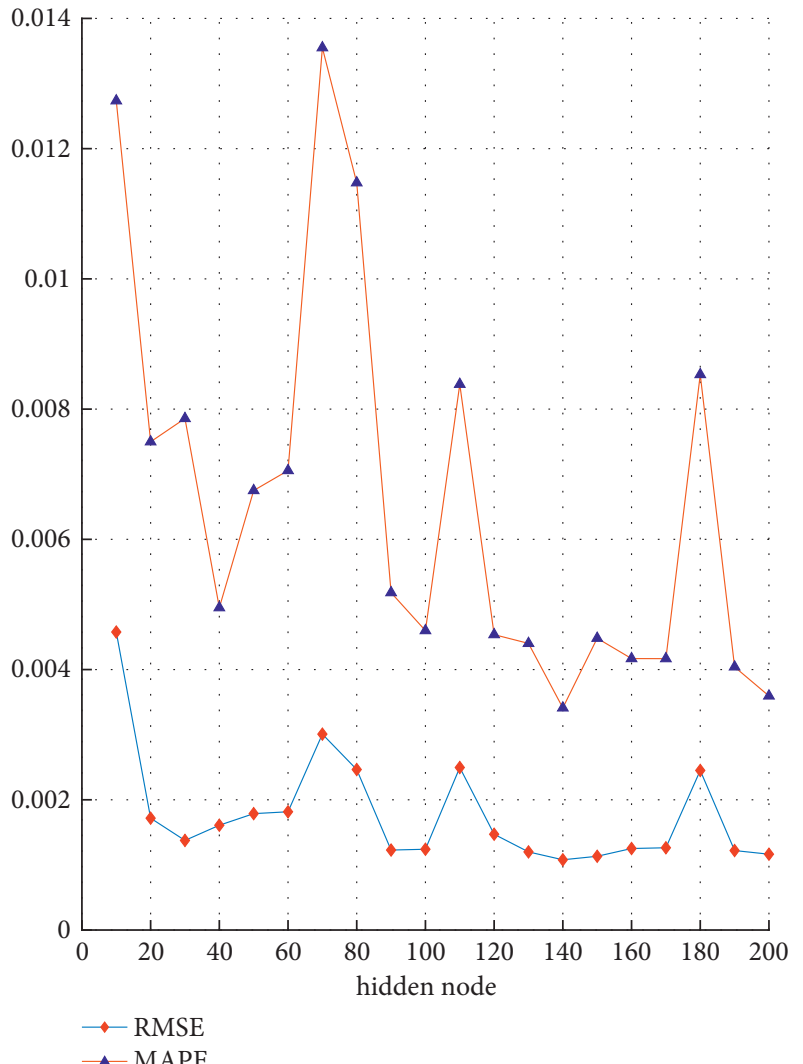

(a)

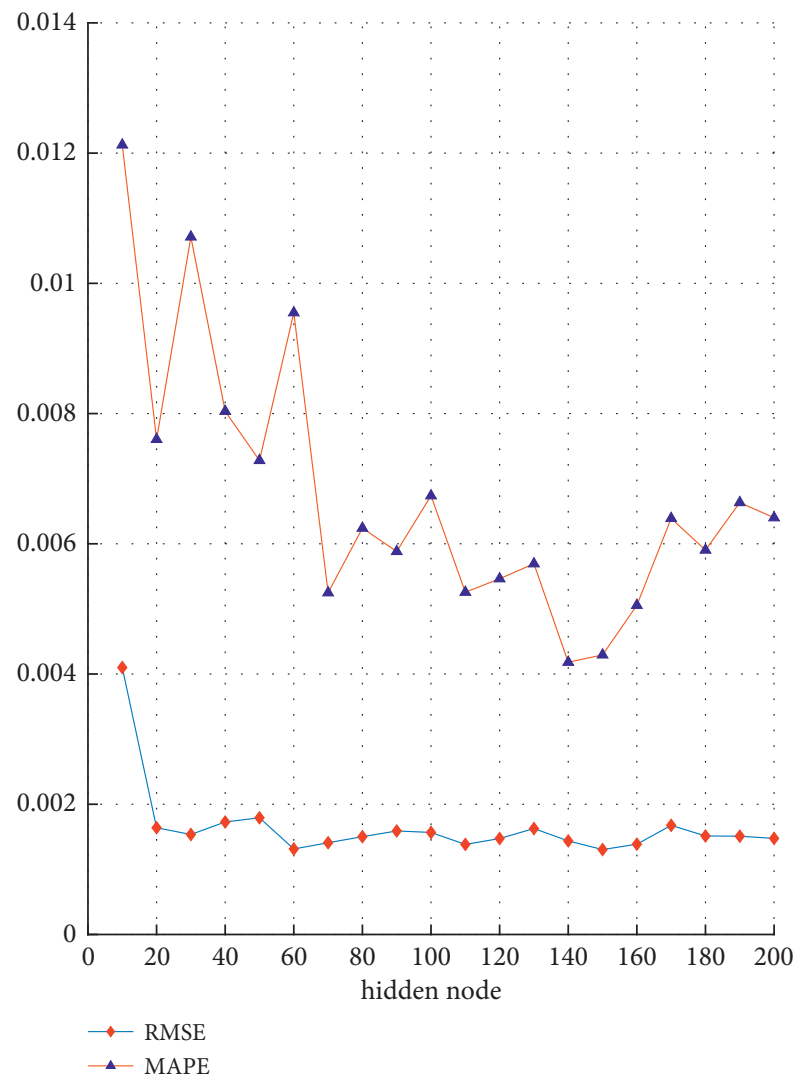

(b)

FIGURE 10: RMSEs and MAPEs of wide and deep model and BPNN model with respect to the number of hidden nodes.

4.2. Neural Network Parameter Selection. This paper selects the experimental data of the 50th charge and discharge as the verification set and the rest as the training set. The backpropagation neural network (BPNN) and wide and deep model are used in this experiment to estimate the SOC. The number of training epochs and the number of hidden neurons in each hidden layer are critical parameters that may influence the performance of the neural network on
SOC estimation. This paper conducts various experiments to select the best parameters of the two models.

The data of battery 1 during charging stage are used to study the influence of these parameters. Firstly, the influence of the number of training epochs on the estimation performance is studied. To be fair, the learning rate and number of hidden neurons are same in the two models. The values of RMSE and MAPE in different training epochs are plotted in 


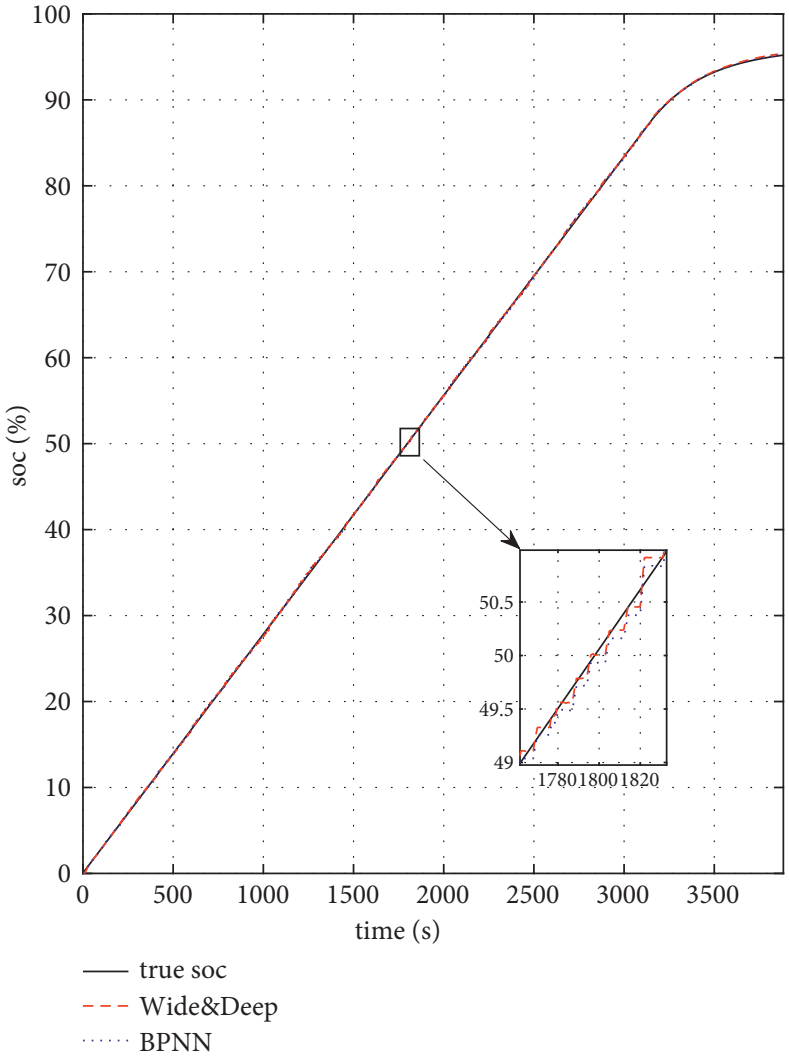

(a)

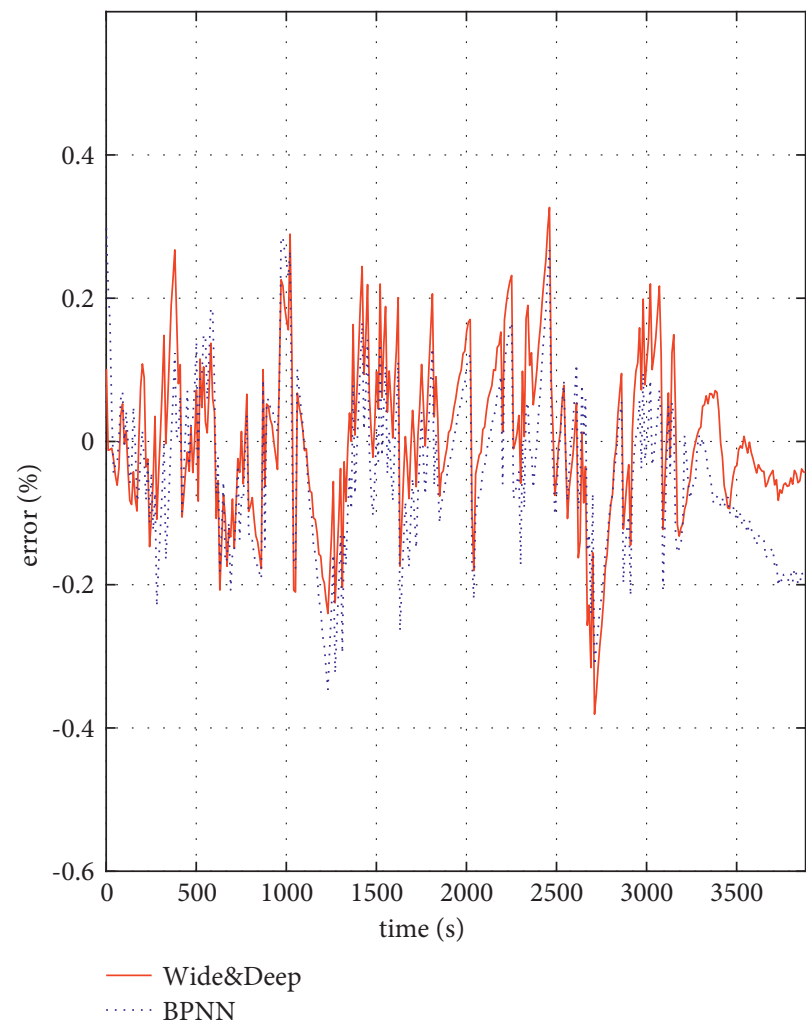

(b)

FIGURE 11: SOC prediction results (a) and errors (b) of battery 1 during charging stage.

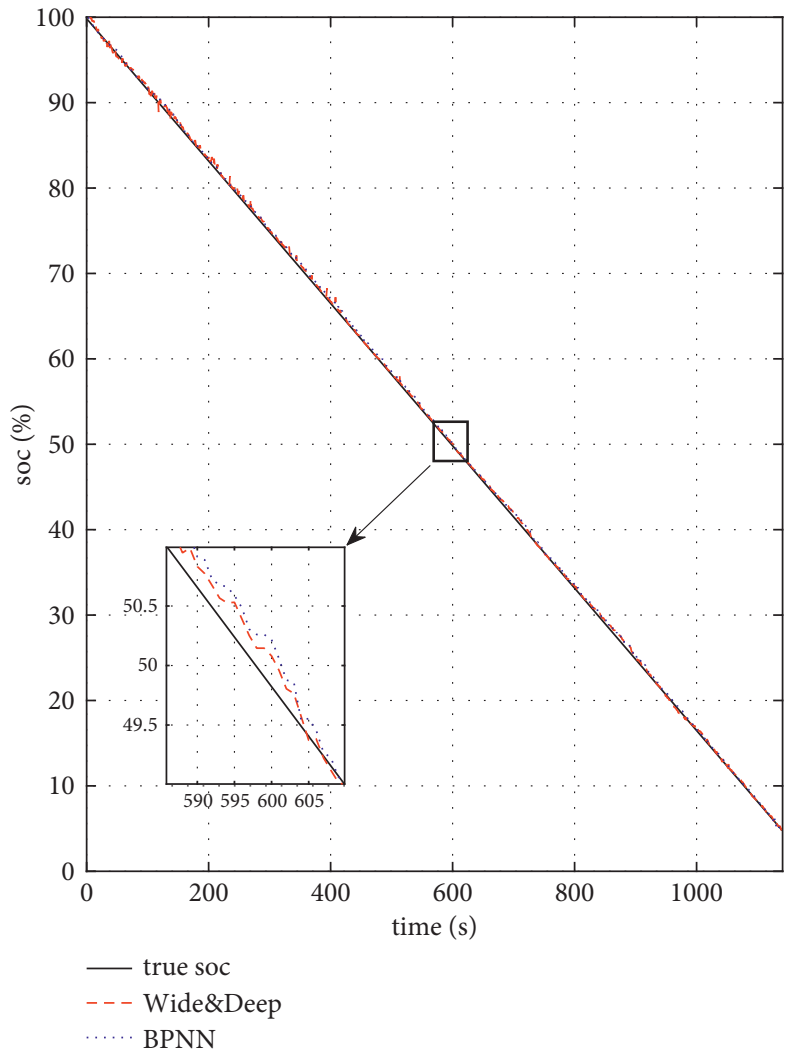

(a)

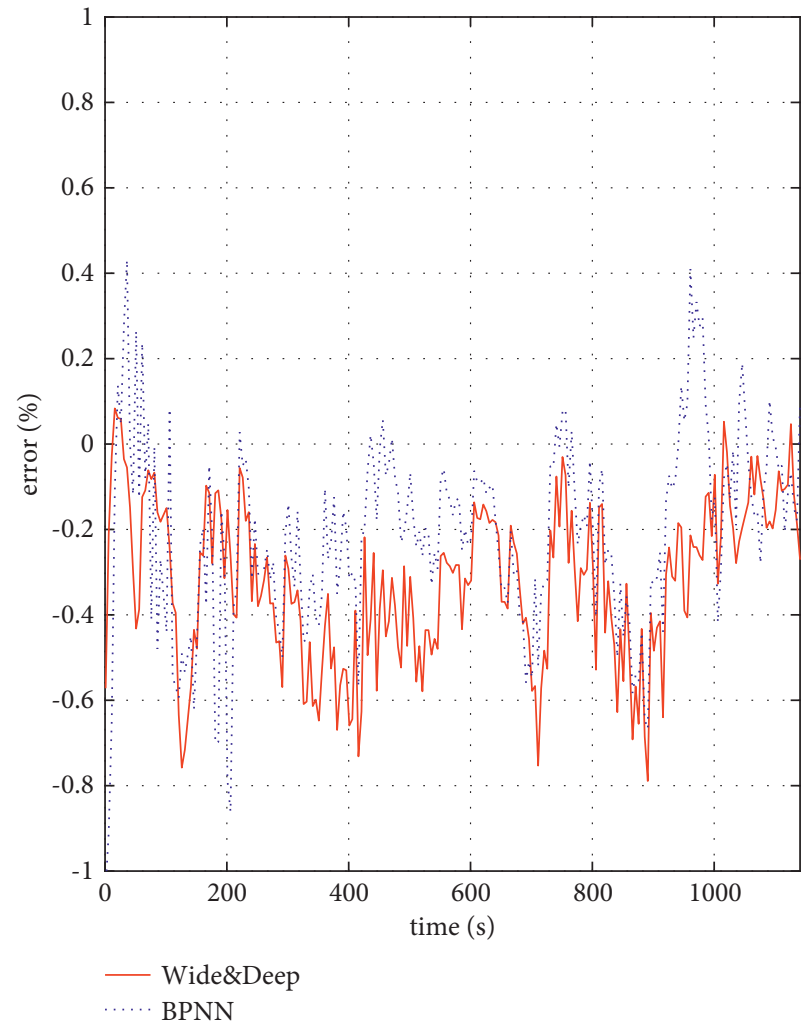

(b)

FIGURE 12: SOC prediction results (a) and errors (b) of battery 1 during discharging stage. 


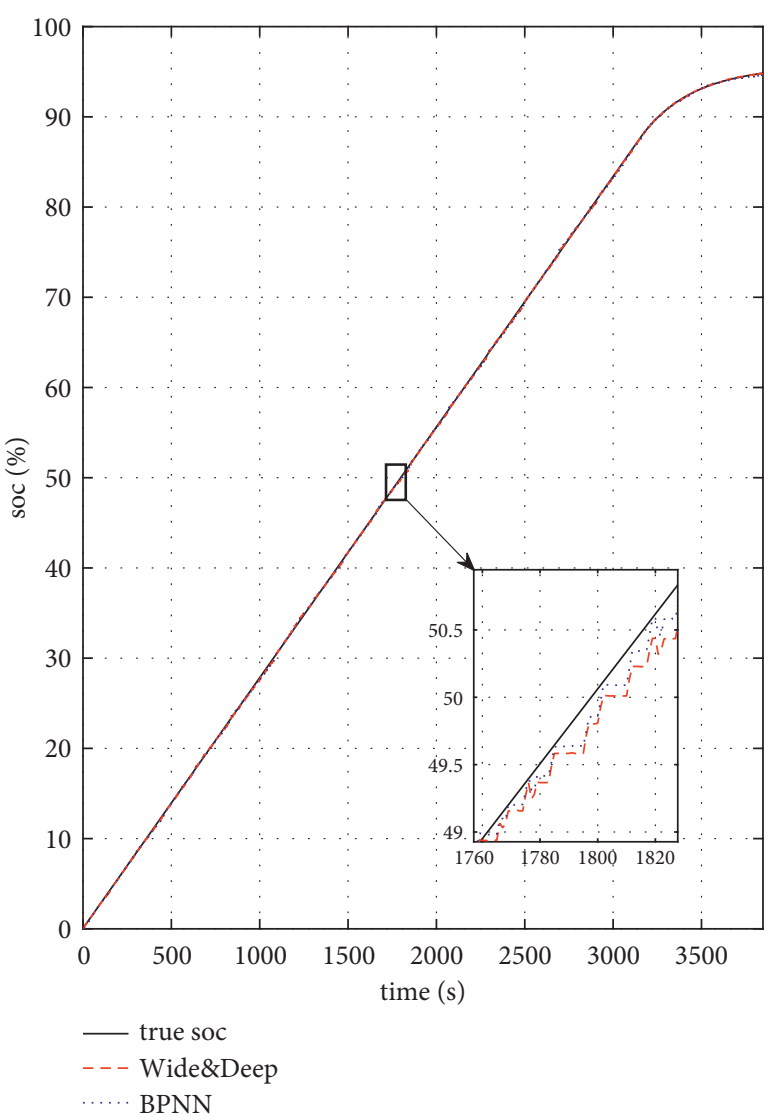

(a)

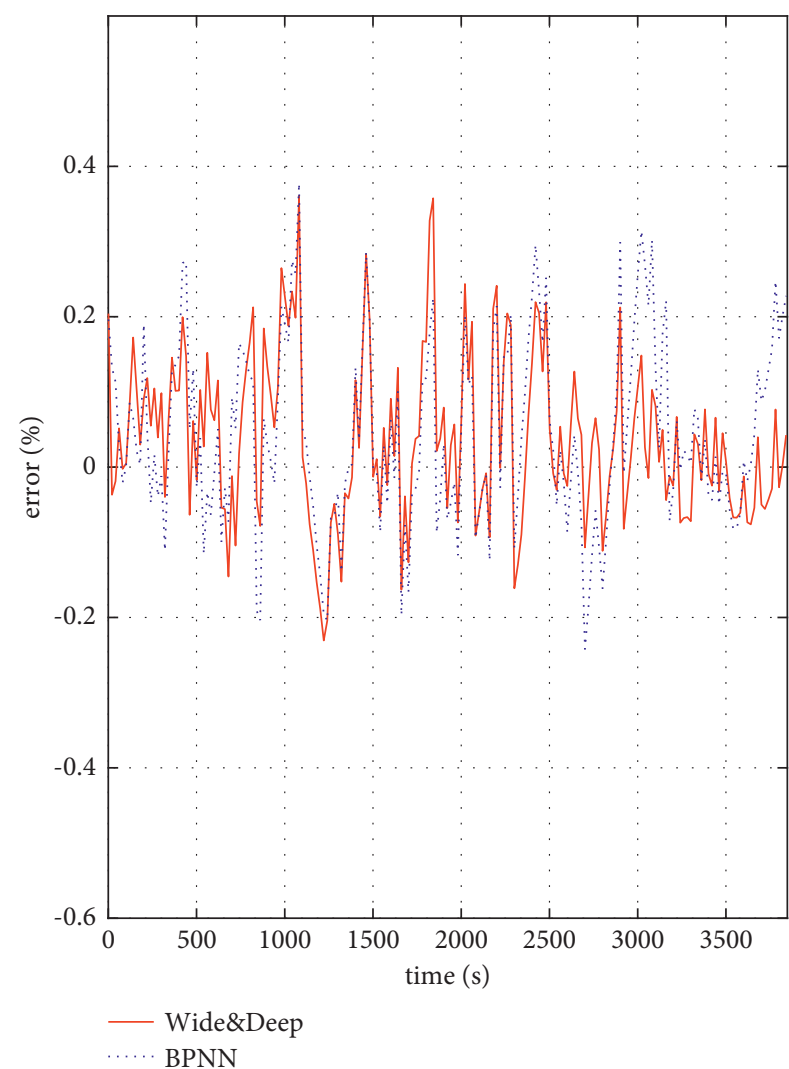

(b)

FIGURE 13: SOC prediction results (a) and errors (b) of battery 2 during charging stage.

Figure 9. In both models, the values of RMSE and MAPE decrease sharply in the first few epochs and remain steady after 50 epochs. Performance after epoch 100 is almost the same. Trading off between testing performance and training time, 100 epochs present an optimal choice.

Next, the performance of the two models is tested under different hidden nodes, ranging from 0 to 200 at intervals of 10. The results are plotted in Figure 10. Generally speaking, using more neurons can better fit the training set, but it will lead to over-fitting problems. On the other hand, too few neurons cannot effectively capture the high-order relationship between input and output. From this perspective, combined with the experimental results, 140 hidden nodes are recommended for the wide and deep model and 150 hidden nodes are recommended for the BPNN model.

4.3. SOC Estimate Results. This paper selects the experimental data of the 50th charge and discharge as the verification set and the rest as the training set. Figures 11-14 show the SOC prediction results of battery 1 and battery 2 at the 50th charge and discharge stage. The solid line in Figures 11(a)-14(a) represents the real SOC value, and the dotted line and point line are the SOC values predicted by the wide and deep model and BPNN model, respectively. Figures 11(b)-14(b) show the errors between the corresponding real SOC and the predicted SOC. It can be seen from the figures that the difference between the SOC predicted by the wide and deep model and the real SOC is very small. The maximum prediction errors of battery 1 during the charging and discharging stage are $0.42 \%$ and $0.86 \%$, respectively. The maximum prediction errors of battery 2 during the charging and discharging stage are $0.50 \%$ and $0.56 \%$, respectively.

4.4. Performance Comparison. This paper uses the common backpropagation neural network (BPNN) to compare various performance indicators with the wide and deep model. Table 2 shows the prediction performance of these two models. Next, the performance of the SOC prediction result of battery 1 is described in detail. Compared with the BPNN model, the RMSE values of the SOC predicted value of the wide and deep model in the charging and discharging stages are reduced by $10.2 \%$ and $15.4 \%$, respectively. The values of MAPE in the charging and discharging stages for the proposed model are improved and reduced by $53.4 \%$ and $26.9 \%$, respectively, in comparison to the common BPNN model. In addition, the SOC error interval of the wide and deep model was smaller than that of the BPNN model. The maximum prediction errors of BPNN model during the charging and discharging stage are $0.50 \%$ and $1.39 \%$, respectively. The maximum prediction errors of the wide and deep model during the charging and discharging stages are $0.42 \%$ and $0.86 \%$, respectively. 


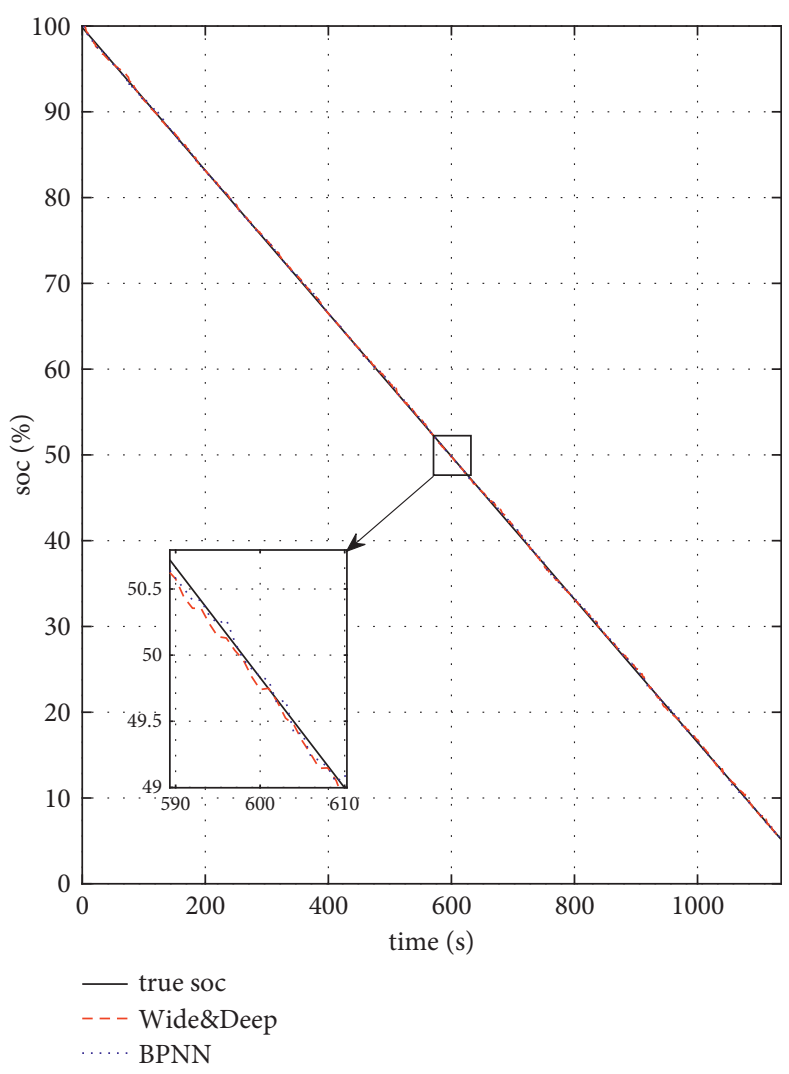

(a)

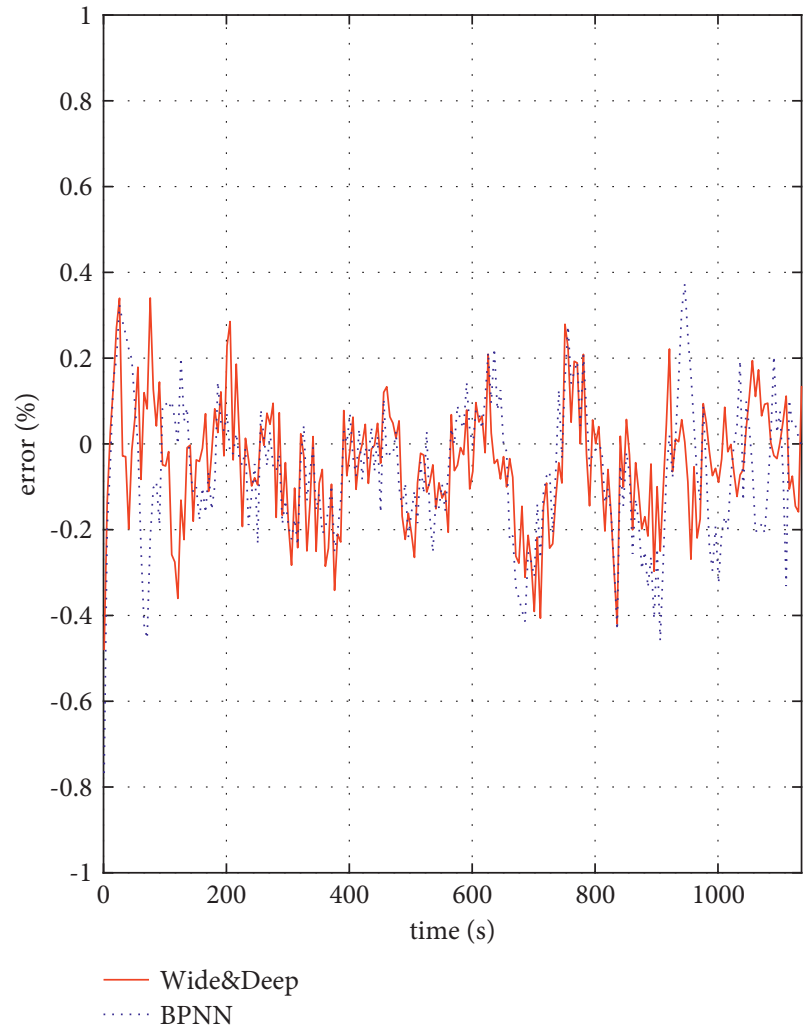

(b)

FIGURE 14: SOC prediction results (a) and errors (b) of battery 2 during discharging stage.

Table 2: Performance comparison between the wide and deep model and BPNN model.

\begin{tabular}{|c|c|c|c|c|}
\hline Model & Stage & $\begin{array}{c}\text { RMSE } \\
(\%)\end{array}$ & $\begin{array}{c}\text { MAPE } \\
(\%)\end{array}$ & Error (\%) \\
\hline \multirow{4}{*}{$\begin{array}{l}\text { Wide and } \\
\text { deep }\end{array}$} & Cell 1 charge & 0.115 & 0.419 & {$[-0.42,+0.38]$} \\
\hline & $\begin{array}{c}\text { Cell } 1 \\
\text { discharge }\end{array}$ & 0.312 & 0.640 & {$[-0.86,+0.38]$} \\
\hline & Cell 2 charge & 0.116 & 0.528 & {$[-0.34,+0.50]$} \\
\hline & $\begin{array}{c}\text { Cell } 2 \\
\text { discharge }\end{array}$ & 0.150 & 0.327 & {$[-0.48,+0.56]$} \\
\hline \multirow{4}{*}{ BPNN } & Cell 1 charge & 0.128 & 0.9 & {$[-0.50,+0.45]$} \\
\hline & $\begin{array}{c}\text { Cell } 1 \\
\text { discharge }\end{array}$ & 0.369 & 0.875 & {$[-1.39,+1.1]$} \\
\hline & Cell 2 charge & 0.131 & 0.848 & {$[-0.41,+0.47]$} \\
\hline & $\begin{array}{c}\text { Cell } 2 \\
\text { discharge }\end{array}$ & 0.171 & 0.412 & {$[-0.77,+0.41]$} \\
\hline
\end{tabular}

\section{Conclusion}

This paper proposes a method to predict the SOC state of lithium-ion batteries based on the wide and deep neural network model. This paper uses the experimental data of 100 times of charging and discharging of two lithium-ion batteries as the training and validation data for the model. The features selected were $i, v, d i, d v, d^{2} i, d^{2} v$, and the number of charging cycles. The wide part of the wide and deep model used a linear regression model, and the input included the original 7 features. The deep part used the BPNN model, and the input was the embedding vector of the 7 original features.

Compared with the common BPNN model, the SOC prediction method proposed in this paper did not consume more time and computing resources, but the accuracy and stability of the prediction were significantly improved. For example, the values of MAPE in the charging and discharging stages for the proposed model are improved and reduced by $53.4 \%$ and $26.9 \%$, respectively, in comparison to the common BPNN model. Compared with the BPNN model, the RMSE values of the SOC predicted value of the wide and deep model in the charging and discharging stages are reduced by $10.2 \%$ and $15.4 \%$, respectively.

However, the data used in this article were obtained under ideal constant temperature conditions, so the model did not take the influence of temperature into account. In addition, the battery always adopted CC-CV charging and $\mathrm{CC}$ discharging protocols, which is rare in real life. In subsequent research, temperature can also be used as a feature, and at the same time, a more realistic charging and discharging protocol can be used to verify the performance of the wide and deep model.

\section{Data Availability}

The data used to support the findings of this study can be accessed by visiting https:/data.mendeley.com/datasets/ c5dxwn6w92/1. 


\section{Conflicts of Interest}

The authors declare that there are no conflicts of interest regarding the publication of this paper.

\section{Acknowledgments}

This study was supported by the State Grid Corporation of China (Research on power grid energy storage management chip technology and prototype development based on neural network) (5700-202055396A-0-0-00).

\section{References}

[1] T. R. Hawkins, B. Singh, G. Majeau-Bettez, and A. H. Strømman, "Comparative environmental life cycle assessment of conventional and electric vehicles," Journal of Industrial Ecology, vol. 17, no. 1, pp. 53-64, 2013.

[2] C. C. Chan, "The state of the art of electric, hybrid, and fuel cell vehicles," Proceedings of the IEEE, vol. 95, no. 4, pp. 704-718, 2007.

[3] J. B. Kerr and B. John, "Advances in lithium-ion batteries," Journal of the American Chemical Society, vol. 125, no. 12, pp. 3670-3671, 2003.

[4] C. She, L. Zhang, Z. Wang, F. Sun, P. Liu, and C. Song, "Battery state of health estimation based on incremental capacity analysis method: synthesizing from cell-level test to real-world application," Journal of Emerging and Selected Topics in Power Electronics, 2021.

[5] L. Zhang, W. Fan, Z. Wang, W. Lib, and Dirk UweSauerb, "Battery heating for lithium-ion batteries based on multi-stage alternative currents," The Journal of Energy Storage, vol. 32, Article ID 101885, 2020.

[6] Z. Wang, C. Song, L. Zhang, Y. Zhao, P. Liu, and D. G. Dorrell, "A data-driven method for battery charging capacity abnormality diagnosis in electric vehicle applications," IEEE Transactions on Transportation Electrification, 2021.

[7] J. Vetter, P. Novák, M. R. Wagner et al., C. Vogler, A. Hammouched, Ageing mechanisms in lithium-ion batteries," Journal of Power Sources, vol. 147, no. 1-2, pp. 269-281, 2005.

[8] S. Piller, M. Perrin, and A. Jossen, "Methods for state-ofcharge determination and their applications," Journal of Power Sources, vol. 96, no. 1, pp. 113-120, 2001.

[9] C. R. Lashway and O. A. Mohammed, "Adaptive battery management and parameter estimation through physicsbased modeling and experimental verification," IEEE Transactions on Transportation Electrification, vol. 2, no. 4, pp. 454-464, 2016.

[10] K. S. Ng, C.-S. Moo, Y.-P. Chen, and Y.-C. Hsieh, "Enhanced coulomb counting method for estimating state-of-charge and state-of-health of lithium-ion batteries," Applied Energy, vol. 86, no. 9, pp. 1506-1511, 2009.

[11] A. Barai, W. D. Widanage, J. Marco, A. McGordon, and P. Jennings, "A study of the open circuit voltage characterization technique and hysteresis assessment of lithium-ion cells," Journal of Power Sources, vol. 295, pp. 99-107, 2015.

[12] G. Dong, J. Wei, and Z. Chen, "Kalman filter for onboard state of charge estimation and peak power capability analysis of lithium-ion batteries," Journal of Power Sources, vol. 328, pp. 615-626, 2016.

[13] H. Pan, Z. Lü, W. Lü, J. Li, and L. Chen, "State of charge estimation of lithium-ion batteries using a grey extended
Kalman filter and a novel open-circuit voltage model," Energy, vol. 138, pp. 764-775, 2017.

[14] S. Ping, M. Ouyang, L. Lu, J. Li, and X. Feng, "The Co-estimation of state of charge, state of health, and state of function for lithium-ion batteries in electric vehicles," IEEE Transactions on Vehicular Technology, vol. 67, no. 1, pp. 92103, 2018.

[15] M. S. Hossain Lipu, M. A. Hannan, A. Hussain, M. H. Saad, A. Ayob, and M. N. Uddin, "Extreme learning machine model for state of charge estimation of lithium-ion battery using gravitational search algorithm," IEEE Transactions on Industry Applications, vol. 55, no. 4, pp. 4225-4234, 2019.

[16] X. Hu, S. E. Li, and Y. Yang, "Advanced machine learning approach for lithium-ion battery state estimation in electric vehicles," IEEE Transactions on Transportation Electrification, vol. 2, no. 2, pp. 140-149, 2017.

[17] Z. Dong, C. Du, H. Lin, C. s. Lai, X. Hu, and S. Duan, "Multichannelmemristive pulse coupled neural network based multi-frame images super-resolution reconstruction algorithm," Journal of Electronics \& Information Technology, vol. 42, no. 4, pp. 835-843, 2020.

[18] J. C. Alvarez Anton, P. J. Garcia Nieto, C. Blanco Viejo, and J. A. Vilan Vilan, "Support vector machines used to estimate the battery state of charge," IEEE Transactions on Power Electronics, vol. 28, no. 12, pp. 5919-5926, 2013.

[19] B. Liu, Y. Yang, Z. Zhao, C. Wu, H. Liu, and Y. Wen, “A batch inheritance extreme learning machine algorithm based on regular optimization," Journal of Electronics \& Information Technology, vol. 42, no. 7, pp. 1734-1742, 2020.

[20] F. Zhou, L. Wang, H. Lin, and Z. Lv, "High accuracy state-ofcharge online estimation of EV/HEV lithium batteries based on Adaptive wavelet neural network," in Proceedings of the Ecce Asia Downunder, pp. 513-517, IEEE, Melbourne, VIC, Australia, June 2013.

[21] L. Gao, Y. Song, and R. A. Dougal, "Wavelet neural network based battery state-of-charge estimation for portable electronics applications," in Proceedings of the The 20th Annual IEEE Applied Power Electronics Conference and Exposition, pp. 998-1002, Austin, TX, USA, March 2005.

[22] S. Tong, J. H. Lacap, and J. W. Park, "Battery state of charge estimation using a load-classifying neural network," Journal of Energy Storage, vol. 7, pp. 236-243, 2016.

[23] L. Kang, X. Zhao, and J. Ma, “A new neural network model for the state-of-charge estimation in the battery degradation process," Applied Energy, vol. 121, pp. 20-27, 2014.

[24] D. Cui, B. Xia, R. Zhang et al., "A novel intelligent method for the state of charge estimation of lithium-ion batteries using a discrete wavelet transform-based wavelet neural network," Energies, vol. 11, no. 4, p. 995, 2018.

[25] M. S. Hossain Lipu, M. A. Hannan, A. Hussain, and M. H. M. Saad, "Optimal BP neural network algorithm for state of charge estimation of lithium-ion battery using PSO with PCA feature selection," Journal of Renewable and Sustainable Energy, vol. 9, no. 6, Article ID 064102, 2017.

[26] H.-T. Cheng, L. Koc, H. Jeremiah et al., "Wide \& deep learning for recommender systems," in Proceedings of the 1st Workshop on Deep Learning for Recommender Systems, pp. 7-10, Boston MA USA, September 2016. 